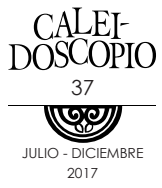

\title{
¿Cómo valoran los adolescentes a la ciencia y a los científicos? Análisis desde una aproximación metodológica mixta
}

\author{
How do adolescents value science and scientists? \\ Analysis through a mixed
}

\author{
FREDI EVERARDO CORREA ROMERO ${ }^{1}$ \\ ALEJANDRA VIRIDIANA ESPINOZA-ROMO² \\ ANDREA DE LOS ÁNGELES VILLANUEVA VALADEZ ${ }^{3}$ \\ TONATIUH GARCÍA CAMPOS ${ }^{4}$ \\ LUIS FELIPE GARCÍA Y BARRAGÁN ${ }^{5}$
}

\section{RESUMEN}

El Programa Internacional de Evaluación de Estudiantes (PISA, por sus siglas en inglés), de la Organización para la Cooperación y el Desarrollo Económicos (OCDE), señala un creciente interés de los adolescentes mexicanos por la ciencia, pero un bajo desempeño en esa área. El Modelo de Probabilidad de Elaboración (MPE) postula que una actitud positiva pero débil no motiva las conductas de las personas. Se realizó un estudio transversal, descriptivo-comparativo con muestreo intencional no probabilístico de 229 adolescentes del estado de Guanajuato. La metodología utilizada fue mixta, incluyendo un estudio cuantitativo con la aplicación de una escala de actitudes y un estudio cualitativo con preguntas abiertas. Los resultados estadísticos muestran una actitud positiva y débil hacia la ciencia, como sugiere el MPE. El análisis de contenido describe a los científicos como inteligentes, aunque aburridos, solitarios y

Doctor en Psicología, profesor en la Universidad de Guanajuato, correafr@gmail.com. Licenciada en Psicología, docente del Departamento de Psicología de la Universidad de Guanajuato, viri.espinozar@gmail.com.

3 Estudiante de Psicología del último semestre en la Universidad de Guanajuato, avival_312@hotmail.com.

4 Doctor en Psicología, profesor en la Universidad de Guanajuato, tonatiuh@ugto.mx.

5 Doctor en Psicología, profesor en la Universidad de Guanajuato, psicosoc@hotmail.com.

$\begin{array}{llllllllllllllll}C & A & L & E & \text { I } & D & O & S & C & O & P & \text { I } & \text { O } & & & \end{array}$ 
locos. Se discute la necesidad de fortalecer las actitudes de los adolescentes hacia la ciencia.

Palabras clave: actitudes; análisis de contenido; ciencia; científicos.

\section{ABSTRACT}

The Programme for International Student Assessment (PISA) of The Organization for Economic Co-operation and Development (OECD) points to a growing interest of Mexican teenagers in science, but underperformance in that area. The Elaboration Probability Model (MPE) postulates that a positive but weak attitude does not motivate people's behavior. The study is cross - sectional, descriptive - comparative with non - probabilistic intentional sampling of 229 adolescents from the state of Guanajuato. The mixed methodology included a quantitative study with the application of a scale of attitudes and a qualitative study with open questions. The statistical results show an attitude towards positive and weak science, as suggested by the MPE. Content analysis describes scientists as intelligent, yet boring, lonely, and crazy. The need to strengthen attitudes towards adolescent science is discussed.

Keywords: attitudes; content analysis; science; scientists.

\section{INTRODUCCIÓN}

De acuerdo con Pérez Tamayo (2010), México presenta un subdesarrollo en el campo científico desde el siglo XIX, en comparación con potencias como Inglaterra, Estados Unidos, Alemania o Francia. De acuerdo con el análisis histórico del autor, la ausencia de dicha tradición científica se debe, en buena medida, a los momentos históricos por los que México ha atravesado, por ejemplo, los acontecimientos de la guerra de Independencia y la Revolución mexicana. Durante esta época el país frenó en distintas ocasiones de manera directa la aparición de varias instituciones que empezaban a desarrollar la tradición científica del país, ejemplo de ello fue el decreto firmado por el presidente Venustiano Carranza en 1915 donde se refiere al Instituto Médico Nacional como no prioritario a los intereses de la nación, con lo que se terminó con una institución fundada en 1888. Algo similar sucedió con el Instituto Patológico Nacional, cerrado dos años antes y 
el Instituto Bacteriológico Nacional, cerrado en 1914. Dichas acciones son evidencia de que el desarrollo científico no figuró entre las prioridades del país, sobre todo durante las repetidas crisis económicas o sociales que México padeció durante los dos últimos siglos (Pérez Tamayo, 2010).

El mismo autor documenta que en la primera mitad del siglo xx se pudo apreciar un cambio significativo en la relación del gobierno en turno con la comunidad científica, ya que se abrieron varios colegios y universidades, pero dichas acciones no desarrollaron una vocación científica entre la población, debido a que los proyectos carecían de una visión a largo plazo. Posteriormente, en la segunda mitad del siglo XX, no se decretó ni sugirió una política nacional de ciencia y tecnología. Los presidentes que tomaron el cargo durante este tiempo prometieron que al final de su sexenio se incrementaría la inversión destinada al desarrollo científico, pero ninguno lo cumplió.

En lo que va del siglo xxi, México ha seguido siendo uno de los países del orbe que menos invierte en su desarrollo científico. La Organización para la Cooperación y el Desarrollo Económicos (OCDE, 2016) reporta que México destinó en el año 2015 sólo 0.6\% del Producto Interno Bruto al desarrollo de la investigación y la ciencia, en contraste con países como Israel, Corea del Sur y Japón, quienes tuvieron una inversión de $4.3 \%, 4.2 \%$ y 3.5\%, respectivamente, en el mismo año (Organización para la Cooperación y el Desarrollo Económicos, 2017).

Adicionalmente a la baja inversión que nuestro país destina para el desarrollo de la ciencia, existe un bajo desempeño de los estudiantes mexicanos de nivel medio superior en comparación con el resto de los países de la OCDE. De acuerdo con el informe del Programa Internacional para la Evaluación de Estudiantes (PISA, por sus siglas en inglés) elaborado por la OCDE (2016), México alcanza 416 puntos de conocimiento en el campo de las ciencias, promedio que se encuentra por debajo del registrado para los miembros del organismo, que es de 493 puntos. Otros países que se hallan a la par junto con México son Colombia y Costa Rica. Asimismo, México se sitúa por debajo de países como Portugal, España, Chile y Uruguay.

Algo similar sucede en la evaluación de matemáticas. Donde el promedio de los estudiantes mexicanos en la prueba PISA fue de 408 puntos, nuevamente por debajo de la media de los países integrantes de la OCDE, que en 2015 fue de 490 puntos. Si bien el promedio de

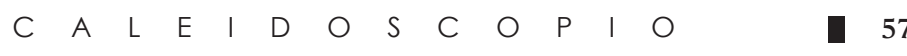


México en esta área había ido aumentando aproximadamente 5 puntos cada tres años, el puntaje de la última evaluación se encuentra por debajo del obtenido durante 2009, que fue de 419 puntos. Adicionalmente, el resultado indica que $23 \%$ de los estudiantes no alcanza el nivel básico de competencia, lo que significa que 1 de cada 4 estudiantes tiene dificultad para identificar cómo una simple situación puede ser representada de manera matemática.

Estos resultados contrastan con las creencias sobre el conocimiento científico que tienen nuestros adolescentes de acuerdo con la misma prueba PISA. Los resultados indican que los estudiantes valoran los conocimientos científicos hacia la investigación, pero también se describe que la disposición de los estudiantes hacia métodos científicos de investigación es menos positiva que lo observado en el promedio de los países miembros de la OCDE. Adicionalmente, el informe señala que se les preguntó a los estudiantes sobre la ocupación que esperaban tener una vez que cumplieran 30 años. En general, 24\% de los estudiantes miembros de la OCDE señala que esperan trabajar en una ocupación que requiera formación científica, mientras que en México resultó que $41 \%$ de los estudiantes tienen dichas expectativas (OCDE, 2016).

Respecto a las diferencias entre hombres y mujeres, los resultados muestran que estas últimas tienden a aspirar a ser profesionales de la salud más que los hombres. Por su parte, una mayor cantidad de hombres tienen la expectativa de trabajar en áreas de tecnologías de la información y comunicación, así como en ingenierías. En México no se registran diferencias significativas entre hombres y mujeres con respecto al nivel de disfrute de las ciencias ni en los niveles de motivación instrumental para estudiar ciencia (OCDE, 2016).

Con base en los antecedentes hasta aquí presentados, podemos concluir que en México hay una escasa tradición científica, a pesar de lo cual, los adolescentes muestran expectativas positivas hacia la ciencia, pero un bajo desempeño que contrasta con esta valoración. Aunque hombres y mujeres eligen diferentes campos científicos, no existen diferencias importantes en su motivación para estudiar ciencia. En términos psicosociales podemos interpretar que existe una evaluación positiva hacia la ciencia pero que no se ve reflejada en conductas que la respalden.

Para comprender la relación entre la valoración positiva y la ausencia de conductas consonantes con dicha valoración, el concepto 
de actitud es un referente ideal, debido a que tiene su origen en las valoraciones que las personas realizan. Adicionalmente, Ajzen (2012) expone que las actitudes son un importante factor que activa y dirige la conducta, por lo que su estudio y comprensión son claves para ayudar a que las valoraciones positivas sobre ciencia que nuestros adolescentes muestran se conviertan en conductas que consoliden una vocación científica a mediano y largo plazos.

Para Petty y Briñol (2012), las actitudes son evaluaciones generales que las personas realizan sobre sí mismas, así como sobre otras personas, grupos, lugares, objetos y situaciones. Por su parte, Eagly y Chaiken (1993), en su definición multicitada, señalan que la actitud es la tendencia psicológica que es expresada a partir de la evaluación particular de alguna entidad con algún nivel de agrado o desagrado. Es decir, se puede evaluar cognitivamente algo de forma agradable (valencia positiva) que aumenta la probabilidad de que la persona realice conductas de aceptación hacia el objeto; por ejemplo, si un estudiante de secundaria tiene una actitud positiva hacia la ciencia, aumenta la probabilidad de tener conductas como realizar las tareas de laboratorio. La evaluación cognitiva que la persona realiza también puede ser de desagrado (valencia negativa), en este caso la conducta es de alejamiento; en nuestro ejemplo, un estudiante con actitud negativa hacia la ciencia considerará aburridas o muy difíciles las tareas del laboratorio, por lo que dejaría de hacerlas. Para que los ejemplos mencionados se reflejen de manera clara en conductas, es necesario que las evaluaciones cognitivas tengan cierto grado de intensidad, por lo que podemos decir que una de las propiedades de las actitudes es su intensidad o fuerza, que va de extrema a débil (Chaiken, 2001). Al aumentar la fuerza de las actitudes, también se incrementa su estabilidad y con ello su posibilidad de activar, dirigir y mantener la conducta de las personas, a diferencia de aquellas actitudes débiles que son más propensas a cambiar a medida que pasa el tiempo (Prislin y Crano, 2010).

De acuerdo con Albarracin, Wang, Li y Noguchi (2010) desde la década de los cincuenta del siglo pasado, Katz y Stotland establecieron que las valencias de toda actitud dependían de tres componentes: el cognitivo, el afectivo y el de comportamiento. El componente cognitivo se refiere a los pensamientos y creencias que la persona desarrolla acerca del objeto evaluado. Por su parte, el componente emotivo o afectivo lo constituyen las emociones y sentimientos que 
son dirigidos al objeto de actitud. Finalmente, el componente de comportamiento o conductual incluye las intenciones o disposiciones a la acción, así como a los comportamientos que sean dirigidos hacia el objeto de actitud (Albarracín et al., 2010).

Existen también indicadores objetivos y subjetivos de la fuerza de las actitudes según señalan Prislin y Crano (2010). Los indicadores objetivos son los que han sido más populares y estudiados; dentro de ellos destacan la extremosidad o polarización, accesibilidad, ambivalencia, resistencia y el potencial predictivo sobre la conducta. Por su parte, los indicadores subjetivos se relacionan con la estimación subjetiva o la manera en que las personas perciben los indicadores objetivos, de ahí la importancia de que las mediciones de las actitudes incluyan estrategias de recolección de datos tanto cuantitativos -por ejemplo, las escalas de actitudes tipo Likert (Morales Vallejo, 2011)-, como cualitativos -por ejemplo, los cuestionarios abiertos- que recojan los pensamientos de las personas sobre el tema en cuestión (Petty y Briñol, 2012). Al respecto, Morales Vallejo (2011) señala en su guía para construir escalas de actitudes que las preguntas abiertas pueden aportar información valiosa y cualitativamente superior que las preguntas de respuesta cerrada, y sugiere la utilización de ambos tipos de preguntas (cerradas y abiertas) para una mejor comprensión de las actitudes.

En la década de los ochenta, Petty y Cacioppo (1986) establecen el proceso a través del cual las personas pueden llegar a manifestar cambios en sus conductas a partir de sus actitudes. El modelo teórico que establece dicho procedimiento fue nombrado como Modelo de Probabilidad de Elaboración (MPE). Petty y Briñol (2012), quienes recolectan los resultados encontrados con el modelo durante 30 años de investigación, indican que cuando una persona recibe un mensaje persuasivo, por ejemplo "es importante estudiar matemáticas", dicha persona analiza el mensaje de manera:

- Racional, a través de la llamada ruta central, que implica una elaboración del grado de acuerdo o desacuerdo con el mensaje persuasivo, tomando como base las creencias, emociones y conductas previas relacionadas con el contenido del mensaje. El resultado de este análisis es una valoración que será coherente con la conducta que la persona realizará en el corto plazo. El mensaje también puede ser analizado de manera: 
- Automática, a través de la llamada ruta periférica, donde la persona utiliza atajos mentales y/o respuestas emocionales que el mensaje persuasivo despierta. El resultado es una conducta inmediata de tipo emocional y producto del momento.

Cuando la motivación y la capacidad que el receptor posee para analizar el mensaje son altas, entonces aumenta la probabilidad de razonamiento o elaboración y con ello actitudes con valencia positiva y de intensidad extrema, pero cuando dicha probabilidad de racionalización es baja, la ruta periférica formará actitudes positivas; en tanto que las de intensidad moderada son menos estables para activar, dirigir y mantener la conducta de las personas (Petty y Briñol, 2012).

Con base en los antecedentes teóricos, se puede establecer una hipótesis de que es probable que exista una actitud positiva, pero de intensidad moderada hacia la ciencia entre una muestra de estudiantes mexicanos. De comprobarse, podría contribuir a explicar los resultados de la prueba PISA 2015 sobre la alta valoración de la ciencia, pero un bajo desempeño. De acuerdo con el mismo informe, podemos suponer que las actitudes de hombres y mujeres son similares, es decir, que el sexo de los estudiantes no influye en sus actitudes hacia la ciencia.

Para verificar esta interpretación, el objetivo de la presente investigación fue conocer las actitudes que tienen hacia la ciencia una muestra de estudiantes (hombres y mujeres) del estado de Guanajuato. Para ahondar más en el significado psicológico de las actitudes se realizó un diseño que incluye mediciones cuantitativas y cualitativas (Morales Vallejo, 2011), que de acuerdo con Kerlinger y Lee (2002), es un modelo mixto de etapas paralelas.

Conocer la actitud de los adolescentes hacia la ciencia nos permitirá proponer estrategias de intervención psicosocial que fomenten la vocación científica, no sólo a través de creencias y expectativas, sino de elementos cognitivos que fomenten la conducta de acercamiento hacia la ciencia dentro y fuera del aula. 
El diseño del estudio es transversal, con alcance descriptivo -comparativo y muestreo intencional no probabilístico y con metodología mixta de etapas paralelas y técnicas de recolección de datos a través de una escala de actitudes y de un cuestionario abierto (Kerlinger y Lee, 2002).

\section{Estudio cuantitativo}

Participantes. Se contó con la colaboración de 229 adolescentes que estudiaban el nivel medio superior, de los cuales $56.3 \%$ corresponde a hombres con una media de edad de 15.83 años (SD $=.58)$ y $43.7 \%$ a mujeres con una media de edad de 15.88 años (SD $=.44$ ), la muestra total de los participantes reportó un promedio de edad de 15.86 años ( $S D=.55)$, todos ellos habitantes del estado de Guanajuato.

Instrumento. Se utilizó la Escala de Actitudes Hacia la Ciencia (Espinoza-Romo, García y Barragán y Correa, 2016), que en su versión original consta de 15 reactivos de formato tipo Likert, con cuatro opciones de respuesta que van desde totalmente en desacuerdo (1) a totalmente de acuerdo (4). El instrumento se creó con el objetivo de evaluar las dimensiones: cognitiva, afectiva y conductual de las actitudes hacia la ciencia.

Procedimiento. La aplicación de la Escala de Actitudes Hacia la Ciencia se realizó en ambientes naturales en el estado de Guanajuato, es decir, en escuelas, parques y plazas. Se solicitó el permiso y la colaboración de los participantes, a quienes previamente se les explicó el propósito del trabajo. Una vez otorgado el consentimiento informado de los participantes, se entregó el instrumento de evaluación y se inició con la fase de respuesta, al término de ésta la escala se entregó al facilitador, se agradeció a los participantes y se finalizó el proceso de levantamiento de datos. Luego de capturar los datos se verificaron los elementos psicométricos de la escala, se realizaron análisis descriptivos y pruebas de comparación de grupos entre hombres y mujeres, con ayuda del programa IBM- SPSS, versión 21. 


\section{Estudio cualitativo}

Participantes. Para la realización del estudio cualitativo participaron 227 estudiantes de nivel medio superior, todos ellos habitantes del estado de Guanajuato; la media de edad que reportaron fue de 15.86 años (SD $=0.53$ ). La muestra estuvo conformada por $46 \%$ de mujeres y $54 \%$ de hombres. Los participantes corresponden a un muestreo no probabilístico por conveniencia.

Instrumento. Para el levantamiento de datos de la metodología cualitativa, se diseñó y aplicó un cuestionario conformado por cinco preguntas abiertas (Hernández, Fernández y Baptista, 2010), las cuales estaban dirigidas a conocer creencias relacionadas con el estudio de la ciencia:

- ¿Qué carrera quieres estudiar? Cuyo objetivo era clasificar el interés vocacional de los estudiantes.

- ¿Qué te gusta leer? El objetivo de la pregunta era conocer el tipo de lectura que realiza por gusto y a partir de ahí deducir su interés por temas científicos

- ¿Es más importante estudiar o ganar dinero? El objetivo de la pregunta era indagar cualitativamente un supuesto que plantea que la ciencia se estudia por vocación más que por interés económico

- ¿Cómo son los científicos? Cuyo objetivo fue conocer las creencias y valoraciones ligadas a la práctica de la ciencia como estilo de vida

- ¿Por qué no te gustaría ser científico? En las pruebas piloto la pregunta anterior tenía claramente respuestas que se podían clasificar como positivas y negativas. Esta pregunta se añadió en el estudio final para explorar la parte negativa de las creencias relacionadas con las personas que se dedican a la ciencia.

Del conjunto de preguntas se presenta el análisis de las dos últimas, debido a que son las que muestran de forma directa las creencias que tiene la muestra sobre las personas que trabajan en ciencia, recordando que, de acuerdo con Ajzen (2012), las creencias son el antecedente que forma la valoración de las actitudes en las personas. 
Adicionalmente, Morales Vallejo (2011) sugiere que un buen análisis de contenido puede dar cuenta de las actitudes en su parte cualitativa con una sola pregunta. El autor menciona que es un procedimiento poco estudiado, debido a la tradición experimental con la que se han estudiado las actitudes, por lo que la inclusión de este tipo de preguntas en un estudio mixto es una contribución metodológica del estudio.

Procedimiento. Con el consentimiento de los participantes e informados sobre el procedimiento y tratamiento de sus datos, se indicó la forma de responder el cuestionario, una vez terminada dicha fase, se agradeció la colaboración de los participantes, asegurando el anonimato y confidencialidad de los datos proporcionados.

Posteriormente, se capturaron todas las respuestas en el programa Microsoft Word 2013 y se asignaron al programa ATLAS.ti versión 7.2, donde a partir de un análisis de contenido se clasificaron las respuestas en códigos emergentes, es decir, se crearon conjuntos de respuestas con contenido similar. Cada conjunto se nombró y se estableció como un código emergente cuyos nombres, definiciones y contenidos eran independientes del resto de los códigos que emergieron del análisis. Posteriormente se volvieron a revisar las respuestas que formaban cada código y se verificó su grado de similitud con el resto de las respuestas. Finalmente, y como lo sugieren Rodríguez, Gil y García (1999), se realizó un microanálisis de contenido para cada pregunta, que se sintetizó en un networks que muestra una descripción esquemática de los códigos. Las relaciones propuestas se basan en el contenido de los códigos emergentes que, a su vez, están formados por las respuestas de las personas agrupadas en temas; es decir, el análisis es producto de la interpretación de las respuestas que los adolescentes aportaron a cada una de las preguntas.

\section{RESULTADOS}

Se siguió el procedimiento de validación propuesto por Reyes y García y Barragán (2008) para determinar las características psicométricas del instrumento, previo a la descripción de los resultados. Luego de verificar que el sesgo y la curtosis de cada reactivo estuvieran dentro del rango de la normalidad estadística, se procedió a verificar la direccionalidad y la capacidad de cada reactivo para discriminar. Se analizó el grado de asociación de los reactivos entre sí y con el total 
para determinar el tipo de rotación utilizada en el análisis factorial. Posteriormente se corrió el análisis factorial a través del método de componentes principales y rotación ortogonal. Se encontró una estructura unifactorial que agrupa los 15 reactivos de la escala. Dicho factor único obtuvo un valor de Kaiser-Meyer-Olkin de adecuación de 0.958 y el valor $\chi^{2}$ para la prueba de esfericidad de Bartlett $=2108 \mathrm{p}$ $<0.05$, que son considerados adecuados y que explican $56.26 \%$ de la varianza. El alfa de Cronbach fue de 0.944. La estructura factorial se puede apreciar en la tabla 1 .

Tabla 1

MATRIZ DE COMPONENTES CON CARGA FACTORIAL

\begin{tabular}{lc}
\hline \multicolumn{1}{c}{ Reactivo } & Carga \\
\hline $\begin{array}{l}\text { Me siento interesado cuando estoy aprendiendo algo } \\
\text { de ciencias }\end{array}$ & 0.844 \\
Me siento bien leyendo sobre cosas de ciencia & 0.842 \\
Me emociona saber cosas de ciencia & 0.838 \\
Pienso que la ciencia es divertida & 0.834 \\
Me siento bien cuando me platican cosas científicas & 0.820 \\
Me entretiene aprender cosas científicas & 0.802 \\
Pienso que leer sobre ciencia me vuelve más interesante & 0.786 \\
Leo cosas que tengan que ver con ciencia & 0.781 \\
Pienso que si aprendo de ciencias me será fácil resolver problemas & \\
en la vida cotidiana & 0.756 \\
Pienso que es bueno aprender de ciencias & 0.6750
\end{tabular}

La estructura unifactorial de la escala contrasta con el plan de prueba que consideraba los tres componentes de la actitud como factores 
independientes. Cuando dicha división se difumina en un factor úni$\mathrm{co}$, se puede suponer que la valoración hacia el objeto es global y no específica, aspecto que se puede explicar en parte por el nivel educativo de la muestra.

En términos descriptivos, la media general fue de 2.85 , con una desviación estándar de 0.675 , recordando que la media teórica es de 2.5. En otras palabras, la actitud hacia la ciencia es ligeramente positiva y con base en los referentes del MPE, dicha actitud es débil y por tanto depende de las emociones que despierte un estímulo o bien de atajos mentales que pueden ser verdaderos o falsos. Otra característica es que dicha actitud es poco estable para dirigir conductas de acercamiento hacia la ciencia por parte de los adolescentes en el mediano y largo plazos.

Como siguiente paso se realizó un análisis de comparación de medias para muestras independientes, con el objetivo de conocer si existen diferencias entre los hombres y las mujeres con respecto a su actitud hacia la ciencia. Los resultados muestran que las mujeres obtienen una media más alta $(\mathrm{M}=2.96)$ que la reportada por los hombres $(M=2.74)$, como lo demuestran los valores de la prueba $t$ de Student: $\mathrm{t}(225)=2.5, \mathrm{p}<0.05$, dichas diferencias resultan estadísticamente significativas.

Para profundizar en la descripción de los resultados y a pesar de que la escala presentó una estructura unifactorial, se crearon tres indicadores con base en el plan de prueba que consideró la evaluación de las dimensiones de la actitud, como lo propone la teoría (Albarracín, Johnson y Zanna, 2005), por lo que se decidió seguir el modelo propuesto teóricamente y analizar por separado cada uno de los componentes. Para el componente cognitivo el promedio fue de 2.92, con una desviación estándar de 0.71. Para el componente afectivo la media fue de 2.92, con una desviación estándar de 0.78. Finalmente, para el componente conductual el promedio fue de 2.68, con una desviación estándar de 0.68 .

Posteriormente se realizó un análisis de comparación de medias para muestras independientes para cada uno de los componentes de la actitud: cognitivo, afectivo y conductual, con respecto al sexo; los resultados muestran diferencias estadísticamente significativas en los componentes cognitivo y afectivo, obteniendo en ambos una media mayor en la muestra de mujeres, en comparación con la media obtenida en la muestra de los hombres (tabla 2). 
Tabla 2

PRUEBA T DE STUDENT PARA MUESTRAS INDEPENDIENTES ENTRE LOS COMPONENTES DE LA ACTITUD HACIA LA CIENCIA PARA CADA SEXO

\begin{tabular}{|c|c|c|c|c|c|}
\hline Componente & Sexo & M & g.1. & $\mathrm{t}$ & $\mathrm{P}$ \\
\hline \multirow[t]{3}{*}{ Cognitivo } & Hombre & 2.79 & & & \\
\hline & & & 225 & 2.921 & 0.004 \\
\hline & Mujer & 3.06 & & & \\
\hline \multirow[t]{3}{*}{ Afectivo } & Hombre & 2.80 & & & \\
\hline & & & 225 & 2.620 & 0.009 \\
\hline & Mujer & 3.07 & & & \\
\hline \multirow[t]{3}{*}{ Conductual } & Hombre & 2.64 & & & \\
\hline & & & 225 & 0.993 & 0.332 \\
\hline & Mujer & 2.73 & & & \\
\hline
\end{tabular}

Nota: $\mathrm{M}=$ media; g.l. = grados de libertad.

La cultura y el lenguaje juegan un rol central en el estudio de cualquier fenómeno social, así como en la búsqueda de explicaciones y soluciones. El discurso es señalado, a menudo, como un lugar donde las creencias, representaciones y actitudes, etc., existen (Santander, 2011 ). Mediante el discurso es posible observar los vínculos y las relaciones que concurren y que conforman un fenómeno, esto como un factor de la construcción de la realidad social.

Dicho lo anterior, con el propósito de enriquecer los datos cuantitativos, se continuó realizando un análisis cualitativo; para ello, a través del microanálisis de contenido de la pregunta ¿cómo son los científicos?, se encontró que existe una representación preponderantemente positiva, obteniendo características asociadas a la inteligencia, la importancia de la ciencia y a la supremacía como elementos recurrentes en el discurso de los jóvenes a quienes se les preguntó; estas mismas características se encontraron vinculadas a otros elementos como la dedicación, la sabiduría y los descubrimientos; lo anterior tejió una red de significados altamente positivos en la representación del científico. Resulta importante señalar la coexistencia de características negativas como, por ejemplo: locos, aburridos y creídos, siendo esta 
última la que mayor peso obtuvo, dado que fue la más frecuentemente mencionada (figura 1).

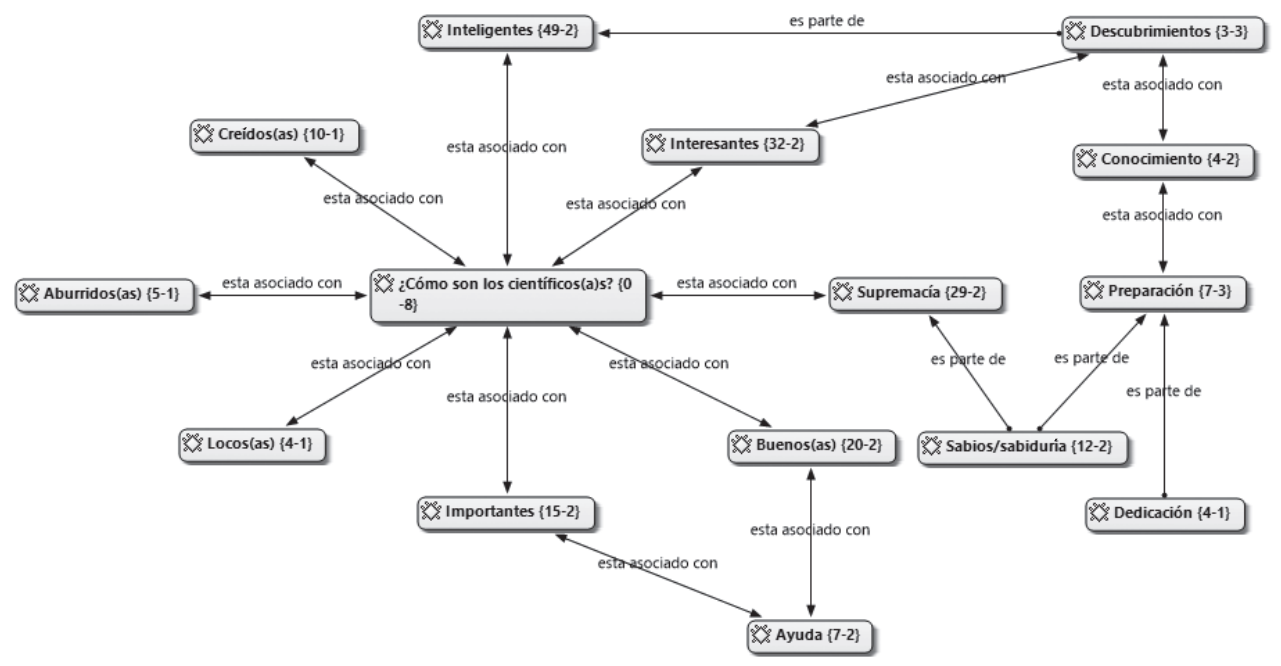

Figura 1. Diagrama explicativo de la pregunta, “¿Cómo son los/as científicos?"

Continuando con los objetivos de investigación, se realizó el análisis de contenido de la pregunta ¿por qué no te gustaría ser científico?, y al igual que en el análisis anterior se encuentra la coexistencia de elementos tanto positivos como negativos, donde destacan la falta de interés y el disgusto, que a su vez se encuentran relacionados. El eje de códigos negativos puede contribuir a explicar por qué las actitudes positivas hacia la ciencia no son tan fuertes, además de aportar elementos para comprender por qué los adolescentes llegan a renunciar a una vocación científica que previamente valoran de forma positiva. Es decir, dado que no se perciben con las habilidades y/o capacidades necesarias para ser científicos, atribuyen al oficio de científico respuestas que se catalogan como aburridas o difíciles (figura 2). 


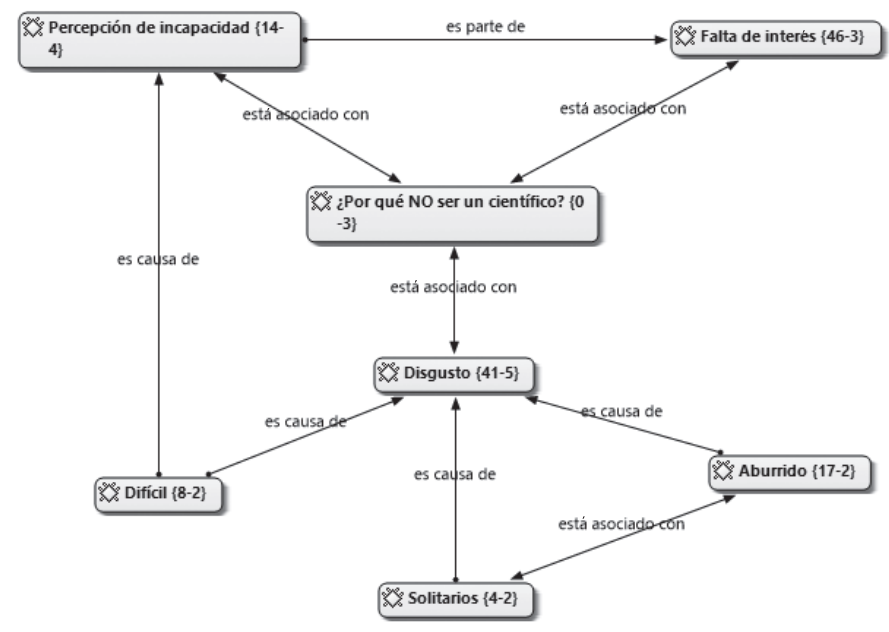

Figura 2. Diagrama explicativo de la pregunta “¿Por qué no te gustaría ser científico/a?”

\section{DISCUSIÓN}

A partir de los resultados del informe PISA de 2015 sobre las valoraciones y expectativas que los estudiantes mexicanos tienen de la ciencia y de los postulados del MPE (Petty y Cacioppo, 1986; Petty y Briñol, 2012), se estableció como supuesto de investigación que los adolescentes mexicanos tienen una actitud positiva, pero con una fuerza débil, lo que disminuye su influencia en conductas académicas que orienten a los estudiantes a cumplir sus expectativas.

Los resultados de la investigación, particularmente los de la etapa cuantitativa, muestran un promedio general y por sexo ligeramente encima de la media teórica, lo que sugiere valencias positivas, pero de mediana fuerza. En ese sentido hay evidencias para respaldar la hipótesis de investigación. Petty y Briñol (2012) señalan que una actitud positiva y débil puede predecir conductas ante situaciones donde se tenga que responder de forma emotiva (por ejemplo, en un estudio de opinión) o con base en heurísticos (las carreras con matemáticas son difíciles). Por el contrario, cuando se habla de vocaciones científicas, debe entenderse que el proceso debe ser más razonado, la decisión de estudiar una carrera científica tiene múltiples elementos a considerar (Chacón y Peña, 2012), por lo que lo ideal sería contar con actitudes 
positivas y fuertes, que sean estables y que, por lo tanto, fomenten procesos de razonamiento ante la toma de decisión de la carrera a elegir.

Se encontraron diferencias por sexo, siendo las mujeres las que tienen una actitud ligeramente más favorable que los hombres hacia la ciencia. Dicho resultado es consistente con lo expuesto en el informe de la OCDE (2016), donde ya se puede observar una tendencia ascendente del número de mujeres que año con año se suman al estudio de algún científico. Sin embargo, es importante aclarar que, aunque nuestros resultados muestran una diferencia estadísticamente significativa, los promedios de ambos grupos están ligeramente encima de la media teórica, es decir, expresan una actitud débil hacia la ciencia.

Al dividir el factor unidimensional en los tres elementos teóricos que conforman una actitud, se encuentra que las mujeres tienen creencias y emociones más positivas hacia la ciencia que los hombres, quizás producto de su creciente incorporación al campo científico y laboral (De la Calle y Rubio, 2010). Sin embargo, el aspecto conductual es igual para hombres y mujeres, el cual es muy cercano a una actitud neutral. Esto sugiere que los estudiantes de nivel medio superior no han tenido experiencias significativas con las distintas ciencias.

Estas suposiciones tienen una confirmación parcial con el análisis de contenido que demuestra que las creencias sobre cómo son los científicos son ambivalentes, por un lado, los adolescentes responden que son personas muy inteligentes, pero por otro lado que son "aburridos", "creídos" y hasta "locos".

De acuerdo con Ajzen (2012), este conflicto entre creencias positivas y negativas contribuye a la debilidad de la actitud y forma una actitud ambivalente que dificulta la medición de la actitud. De ahí la trascendencia de utilizar una metodología mixta y tener indicios de las creencias a través de preguntas abiertas.

Ante la cuestión sobre si es posible analizar una actitud a través de una sola pregunta, Morales Vallejo (2011) señala que, evidentemente, depende de la pregunta y la cantidad de información que la muestra aporte. Señala que el riesgo es perder validez de constructo. Al respecto, Kvale (2011) indica que el análisis de contenido realizado a entrevistas estructuradas o cuestionarios abiertos no es válido si se limita dicho concepto a un número; sin embargo, al ampliar el concepto de validez a la precisión en que nuestras evaluaciones reflejan la realidad es posible alcanzar dicha validez a través del análisis cualitativo de una o varias preguntas. Finalmente, Kerlinger y Lee (2002) evaden esta 
discusión, pero señalan que la utilización de métodos cuantitativos que ofrecen pruebas de validez puede complementarse con los resultados del análisis cualitativo que profundizan en aquellos aspectos que escapan a su contraparte cuantitativa.

A la pregunta de, ¿por qué no ser un científico?, se mencionan respuestas como aburrido, solitario, difícil, falta de interés e incapacidad. La cuestión de la incapacidad puede interpretarse, de acuerdo con Bandura (2012), como una baja autoeficacia en ciencias. Esto puede contribuir a explicar el bajo desempeño que los adolescentes presentan en matemáticas (Rodríguez, 2011) y que, como se pudo observar, en el caso de México sigue estando por debajo del promedio de los miembros de la OCDE.

Si pensamos en el interés como una necesidad psicológica de las personas (Damasio, 2013) ligada a la experiencia, los resultados de la investigación sugieren que hace falta "vivir" más la ciencia, experimentarla y, con ello, fomentar este elemento.

Dentro del análisis histórico que Pérez Tamayo (2010) realiza de la ciencia en México, destaca que uno de los principales puntos a favor que se viven actualmente es que el grupo social conformado por estudiantes y egresados con estudios universitarios, así como el número de académicos, ha crecido de manera importante durante los últimos años, lo cual influye fuertemente en la idea de que la ciencia es indispensable para el desarrollo cultural, social y económico de México.

Nieda y Macedo (1997) señalan que la cultura científica ha ido ganando lugar dentro de la sociedad en diferentes temas como la salud, los recursos alimenticios y energéticos, la conservación del medio ambiente, el transporte y los medios de comunicación, así como en las condiciones que mejoran la calidad de vida del ser humano.

Por su parte, Chácon y Peña (2012) señalan que impulsar el gusto tanto por la vocación como por la educación científica es un compromiso que involucra al gobierno, a la ciudadanía y al sector privado.

Los resultados aquí planteados sugieren que desde la psicología se deben plantear estrategias de intervención que reduzcan los estereotipos negativos hacia los científicos, además de contribuir a formar actitudes positivas hacia la ciencia que sean fuertes y mejorar la autoeficacia científica que actualmente tienen los adolescentes.

Una limitación del estudio en general fue que se analizó la actitud de la ciencia en general y en el estudio cualitativo se habló de los científicos, sin embargo, una investigación sobre disciplinas específicas 
podría complementar los resultados que se destacan en la presente investigación.

Finalmente, Bauman (2009) señala que en los tiempos modernos la autogestión del conocimiento es fundamental, debido a que el conocimiento está a un clic de distancia y, sin embargo, los estudiantes utilizan esa herramienta con fines de entretenimiento. Desde la psicología se pueden y deben desarrollar estrategias de intervención que fomenten la autodeterminación y su orientación al conocimiento (Deci y Ryan, 2012), para ello, una actitud positiva y razonada es una base excelente para guiar los intereses de los estudiantes y motivarlos a ser los futuros científicos que el país necesita.

\section{REFERENCIAS}

Ajzen, I. (2012). The theory of planned behavior. En P. A. M. Van Lange, A. W. Kruglanski y E. T. Higgins (Eds.), Handbook of Theories of Social Psychology (pp. 438-459). New York: Sage.

Albarracín, D., Johnson, B. y Zanna, M. (2005). Attitudes: introduction and scope. The Handbook of Attitudes (p. 4). New Jersey: Lawrence Erlbaum Associates.

Albarracín, D., Wang, W., Li, H. y Noguchi, K. (2010). Attitude measurement. En W. D. Crano y R. Prislin (eds.), Attitudes and Attitud Change: Frontiers of Social Psychology (pp.19-40). New York: Psychology Press.

Bandura, A. (2012). Social cognitive theory. En P. A. M. Van Lange, A. W. Kruglanski y E. T. Higgins (Eds.), Handbook of Theories of Social Psychology (pp. 349-374). New York: Sage.

Bauman, Z. (2009). Los retos de la educación en la modernidad líquida. Barcelona: Gedisa.

Briñol, P. y Petty, R. E. (2012). The history of attitudes and persuasion research. En A. Kiglansky y W. Stroebe (Eds.), Handbook of the History of Social Psichology (pp. 285-320). New York: Psychology Press.

Chacón, A. y Peña, P. (2012). Cómo cambiar historias. Lo que podemos hacer los individuos, las empresas y las organizaciones sin fines de lucro por la educación en México. México, D.F.: Fondo de Cultura Económica.

Chaiken, S. (2001). Attitude Formation: Function and Structure. En N. J. Smelser y P. B. Baltes (Eds.), International Encyclopedia of the Social \& Behavioral Sciences (pp. 899 - 905). Oxford: Pergamon. 
Damasio, A. R. (2013). En busca de Spinoza. Neurobiología de la emoción y los sentimientos. España: Paidós.

De la Calle, L. y Rubio, L. (2010). Clasemediero. Pobre no más, desarrollado aún no. México: CIDAC.

Deci, E. L. y Ryan, R. M. (2012). Self-Determination theory. En P. A. M. Van Lange, A. W. Kruglanski y E. T. Higgins (Eds.), Handbook of Theories of Social Psychology (pp. 416-437). New York: Sage.

Espinoza-Romo, A. V., García y Barragán, L. F. y Correa, F. E. (2016). Medición de las actitudes hacia la ciencia. Lógica, teoría de la argumentación y filosofía de la ciencia. Ponencia llevada a cabo en el 5 to Congreso Internacional de Ciencias Sociales en el Sureste Mexicano. Octubre de 2016, Cancún (México).

Kerlinger, F. N. y Lee, H. B. (2002). Investigación del comportamiento: Métodos de investigación en Ciencias Sociales. México: McGraw-Hill.

Kvale. S. (2011). Las entrevistas en investigación cualitativa. Madrid: Morata Morales Vallejo, P. (2011). Construcción de escalas de actitudes tipo Likert. Madrid: La Muralla.

Morales, F., Gavira, E., Moya, M. y Cuadrado, I. (2007). Actitudes, cambio de actitudes y procesos de influencia. En Manuel Cejudo (Eds), Psicología Social (pp. 457-587). Madrid: McGraw Hill.

Nieda, J. y Macedo, B. (1997). Un Currículo Científico para Estudiantes de 11 a 14 años. Madrid: OEI - UNESCO/Santiago. Recuperado de: http:// campus-oei.org/oeivirt/curricie/curri01.htm.

Organización para la Cooperación y el Desarrollo Económicos (2016). Programa para la Evaluación Internacional de Alumnos (PISA) PISA 2015 - Resultados [México]. Recuperado de: https://www.oecd.org/pisa/ PISA-2015-México-ESP.pdf.

Organización para la Cooperación y el Desarrollo Económicos (2017). Compare su país - Science and Technology Indicators. Recuperado el 7 de marzo de 2017, de: http://www.compareyourcountry.org/science-and-technology?cr=mexElg=esEpage $=0$ Evisited $=1$.

Pérez Tamayo, R. (2010). El estado y la ciencia en México: pasado, presente y futuro. En H. Fix-Zamudio y D. Valadés (Eds.), Formación y perspectivas del Estado en México. (pp. 319-349). México: UNAM - El Colegio Nacional.

Petty, R. y Krosnick, J. (1995). Attitude strength: Antecedents and consequences. New York, NY: Erlbaum. 
Petty, R. E. y Briñol, P. (2012). The elaboration likelihood model. En P. A. M. Van Lange, A. W. Kruglanski y E. T. Higgins (Eds.), Handbook of Theories of Social Psychology (pp. 224-245). New York: Sage.

Petty, R. y Cacioppo, J. (1986). The elaboration likelihood model of persuassion. Advances in Experimental Social Psychology, 19, 123-205. doi: 10.1016/S0065-2601(08)60214-2.

Prislin, R. y Crano, W. D. (2010). Attitudes and attitude change: the fourth peak. En W. D. Crano y R. Prislin (Eds.), Attitudes and Attitud Change: Frontiers os Social Psychology (pp. 3-18). New York: Psychology Press.

Reyes Lagunes, I. y García y Barragán L. F. (2008). Procedimiento de validación psicométrica culturalmente relevante: un ejemplo. En S. Rivera Aragón, R. Díaz-Loving, R. Sánchez Aragón, I. Reyes Lagunes (Eds.) La psicología social en México. (pp. 625 - 630) México: AMEPSO.

Rodríguez, G., Gil, F. y García, J. (1999). Metodología de la investigación cualitativa. Málaga: Ediciones Aljibe.

Rodríguez, M. (2011). La matemática y su relación con las ciencias como recurso pedagógico. Revista de Didáctica de las Matemáticas, 77, pp.35-49. Recuperado de: http://www.sinewton.org/numeros/numeros/77/Articulos_01.pdf.

Santander, P. (2011). Por qué y cómo hacer análisis del discurso. Cinta de Moebio: Revista de Epistemología de Ciencias Sociales, 41, 207-224. doi: 10.4067/S0717-554X2011000200006. 